\title{
Forecasting COVID-19 Pandemic in India and its Impact on Planet, People and Profit
}

\author{
${ }^{1}$ Om Patra ${ }^{*},{ }^{2}$ Dinesh Darshan, ${ }^{3}$ Elizabeth Abba \\ ${ }^{1,2}$ Xavier School of Computer Science \& Engineering, Xavier University Bhubaneswar \\ ${ }^{3}$ Associate Professor, Xavier School of Sustainability, Xavier University Bhubaneswar
}

\begin{abstract}
The outbreak of coronavirus disease 2019 (COVID-19) has created a global health crisis that has had a deep impact on humanity. The only safety measures put in place to contain the spread of the virus require social distancing which led to nationwide lockdown. Now the global health crisis is advancing to a situation of global economic crisis. Some of the greatest countries with stable and secure economic conditions are getting edgy and stressed out due to the spread and heavily contagious nature of the Coronavirus disease. Due to the increasing number of daily cases it is a necessity to develop a prediction method. This paper aims at predicting the number of Coronavirus cases in India ahead of a month and also predicting individually some specific states/UT of India. The paper will also elucidate the indirect effects of lockdown in India. This section will shed light on the indirect effects of the lockdown on people, planet and profit during the lockdown period taking account of the situation in India. This detailed annotation will build a foundation for the people of India to be prepared for upcoming days.
\end{abstract}

\section{Introduction}

On 31st December 2019, The World Health Organization (WHO) was informed regarding a few instances of pneumonia of obscure reason in Wuhan City, China. A novel coronavirus was distinguished as the reason by Chinese experts on 7 January 2020 and was incidentally named "2019-nCoV" [1].On February 11, 2020, this pestilence was formally named as COVID-19 and was recognized as an irresistible infection bringing about general wellbeing crisis, as it immediately spread inside China and to assist 24 nations which are arranged topographically between $42.937084^{\circ} \mathrm{N}$ and $-75.6107^{\circ} \mathrm{E}$ [2] and in this manner spreading to the whole world. The novel coronavirus has become a global public health problem. The significant contrast between the pandemic brought about by CoV-2 and related infections, as Severe Acute Respiratory Syndrome (SARS) and Middle East Respiratory Syndrome (MERS), is the capacity of CoV-2 to spread quickly through human contact and leave almost $20 \%$ tainted subjects as symptomless bearers [3]. The older individuals and patients with comorbidities are increasingly powerless against extreme impacts of COVID19[4]. In the absence of any therapeutic drug most countries have tried to mitigate the spread of the virus with massive COVID-19 screening tests and by strictly implementing "social distancing" to break the chain of spread of the highly contagious virus and also making people aware of the unseen danger and suggesting them to wash and sanitize their hands frequently and other ways to maintain a personal hygiene.

India, the second most populated country in the world implemented the public policy of social distancing and strictly implemented nationwide lockdown and terminating all kinds of movement among people. Later on, implemented the containment zone model strictly to contain the spread of the disease. But due to some unforeseen circumstances there was movement among people to return to their hometown which added fuel to the fire of spreading the disease.

The lockdown is a great step for containing the disease at one place, but it is severely affecting the poor and migrant laborers who depend upon daily wages. Staying indoors without any work or more precisely without any wage is not going to end well. This situation of crisis and its effects upon the general mass and individuals are reported by the media all over the world. Many researches and investigations are being carried out at all levels to quickly assemble information and data, create moderation strategies and 
implementation of the equivalent [5]. The pandemic has likewise set off a worldwide economic crisis, whose shock will be felt for quite a long time to come. As of now due to the widespread larger part of our habits, exchange, social and financial relations, strategies, shapes of work, and political organizations have as of now in a general sense changed [6]. The administration and the health authority are putting their all efforts on the treatment of the symptomatic patients and tracing their contacts to further isolate them. To address Covid-19 ahead of time requires a prediction tool to understand the number of cases rising in future so that administration can be better prepared to take action [7].

Machine Learning techniques and tools [8] can be utilized to handle large data and intelligently foresee the spread of the disease. This paper here considers and discusses the proposed prediction models of COVID-19 spread in India as well as some states/UTs like Maharashtra, Delhi, West Bengal, Tamil Nadu and Odisha using Gated Recurrent Unit (GRU) [9] and Facebook's Prophet [10]. These models will predict the number of confirmed cases in India and the states mentioned one month ahead i.e. till 1st July 2020.

One the other side this lockdown period has also provided ample opportunities to the environment to restore. The shutting down of industries, factories and reduction of all kinds of vehicles will help in the renewal of nature and its elements. In this period, it is also observed that there is a better air quality in different parts of the world [[11]. At last this paper will elucidate the indirect effects of lockdown in India. Under this the cynosure is the changing trend of people, planet and profit before the implementation of lockdown and during the lockdown period. This detailed annotation of the entire situation in India will build a foundation for the people and the administration to be prepared for upcoming days.

\section{Preliminaries}

This section summarizes the different techniques used in this paper to forecast COVID-19 cases in India, which includes GRU, Facebook's Prophet.

\subsection{Gated Recurrent Unit (GRU)}

GRU is a special type of Recurrent Neural Network to handle the vanishing gradient problems [12] and exploding gradients in traditional RNNs when learning long-term dependencies. GRU was introduced in 1997 and further improved over the next few years [13]. Along with the long short-term memory (LSTM) unit [14], GRU is one of the most extensively used models in deep learning for sequence data problems. According to [15], GRU cells don't have isolated memory cells like the architectural design of the LSTM cell. In addition, the GRU has only two gate layers, i.e. reset gate, and update gate rather than three gate layers in each module like LSTM. The reset gate decides how much data you should forget from past memory. The update gate is acting like an LSTM cells forget and input gate. This decides how many data can be transferred on to the future from past memory.

\subsection{Facebook's Prophet}

PROPHET is made public via the Core Data Science team on Facebook. Prophet based on an additive model where non-linear patterns are equipped with annual, monthly, and regular seasonality. PROPHET architecture has their unique data frame for efficient time series and seasonal handling. The data frame is composed of two columns of foundations. One such column is ds; and in this column it stores a time series of dates. The second column is y; and the corresponding time series values are stored in the data frame. Therefore, the system operates smoothly on a seasonal time series and offers some options to manage the seasonality of the datasets. As these choices are presented, the data analyst will choose the time granularity for the forecast model in the datasets. It works best with time series that have clear seasonal effects and historical seasonal records. The Prophet is resilient to missing trend data and transformations, and it also manages outliers. [16]

\section{Methodology}

This study analyzes the effectiveness of our prediction approach using COVID-19 data for the experimental application. The data set for daily cases of COVID-19 in India and state wise data is collected from the Center for Systems Science and Engineering (CSSE) at Johns Hopkins University and https:/www.covid19india.org/respectively. The COVID-19 data used in this study includes the daily number of confirmed, recovered, and deaths from March 2020 through June 2020. The training set consists of the 
first 80 percent of the sample, while the remaining 20 percent has been used as the test set to avoid overfitting during training.

\section{Experimental Approach}

The data was standardized to improve performance as LSTM, GRU, and PROPHET comparators. The sample data set was divided into two datafiles to start model building — Training (80\%) and Testing (20\%). The withheld test dataset was used only to check the efficiency of the built classification models. This approach brings some insight into model success in real-world environments.

\section{Performance Evaluation}

\begin{tabular}{|l|l|l|}
\hline Model & RMSE & R2 \\
\hline Prophet & 3514.764 & 0.97845 \\
\hline LSTM & 2988.845 & 0.99588 \\
\hline GRU & $\mathbf{2 5 6 5 . 2 5 7}$ & $\mathbf{0 . 9 9 6 9 7}$ \\
\hline
\end{tabular}

Table 1: Calculated Value of RMSE (Root Mean Squared Error), R2 (R-Squared Error)

The performance parameters of the model are then assessed to test for reliability in predicting the outcome. Root mean square error (RMSE), R2 is calculated and shown in Table 1. The results show that the model performed well in fitting the cumulative cases in India while a relatively weak fitting is observed in the daily number of cases for different states i.e. Maharashtra, Tamil Nadu, Delhi, West Bengal, Odisha. The daily data show that there are many spikes which reduces the accuracy of predictability of the model. The result obtained was contrasted with LSTM and Prophet, and the Gated Recurrent Unit (GRU) was found to outperform current models and the accuracy and performance recorded on the benchmark data sets have increased significantly in recent times.

\section{Result Analysis}

(a)

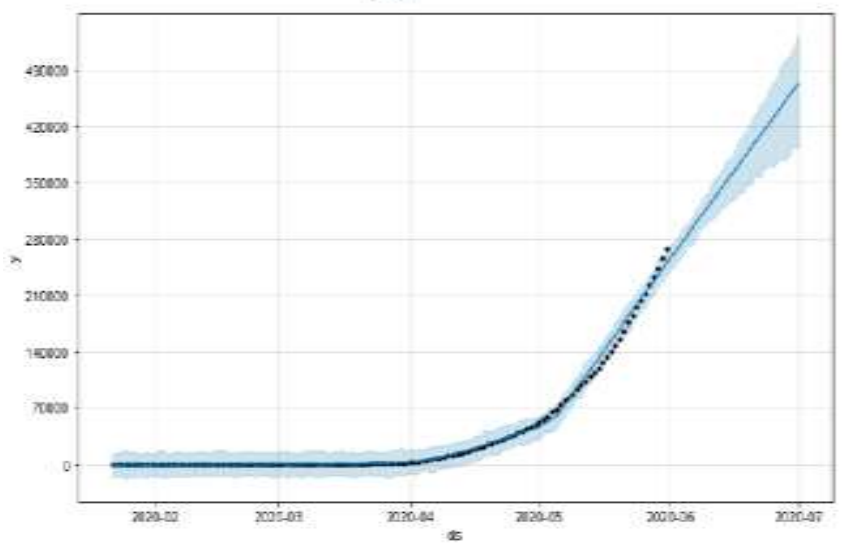

(b)

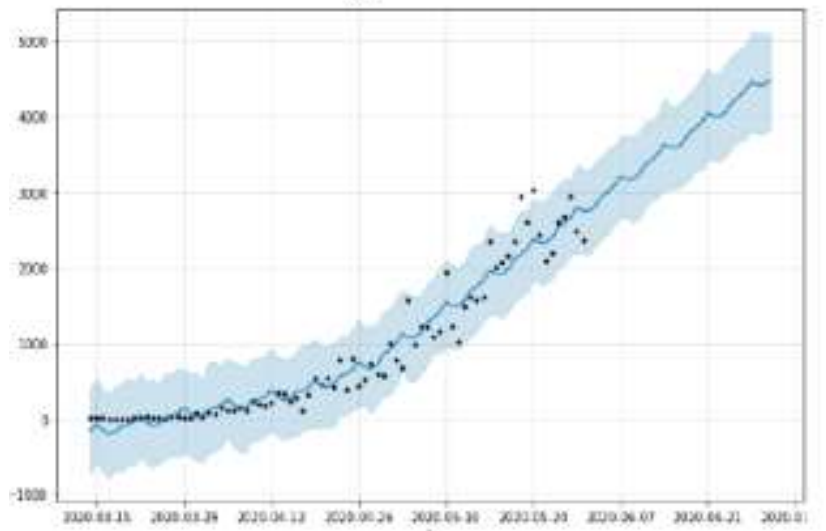

(d)

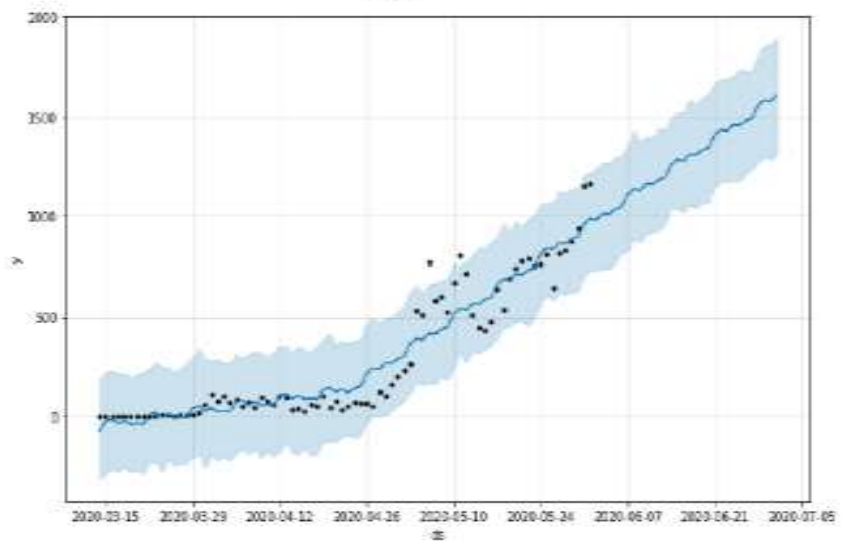

(e)

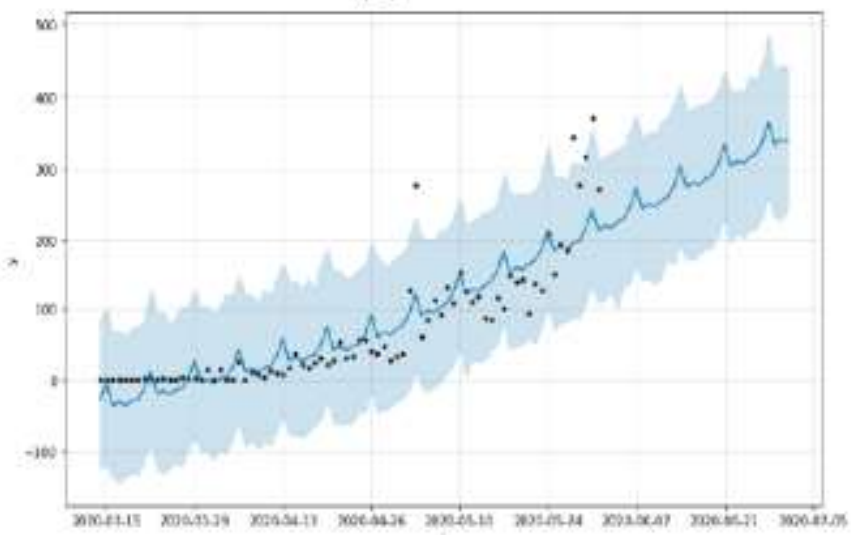


(c)

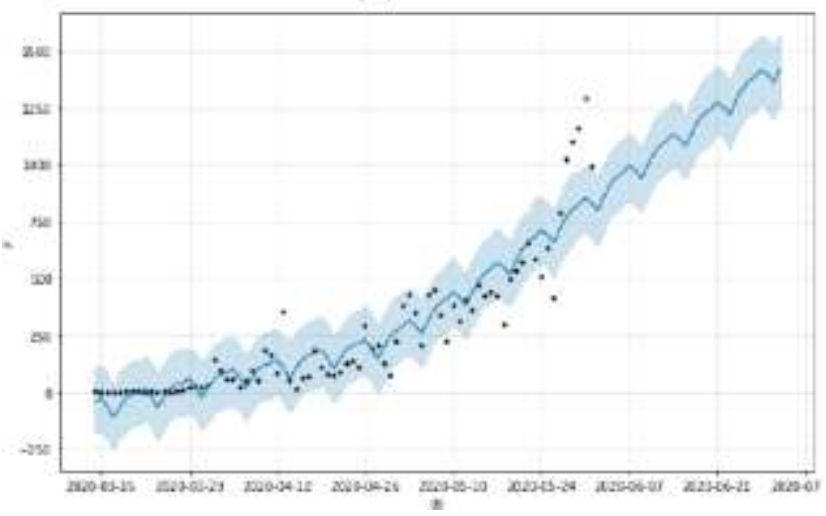

(f)

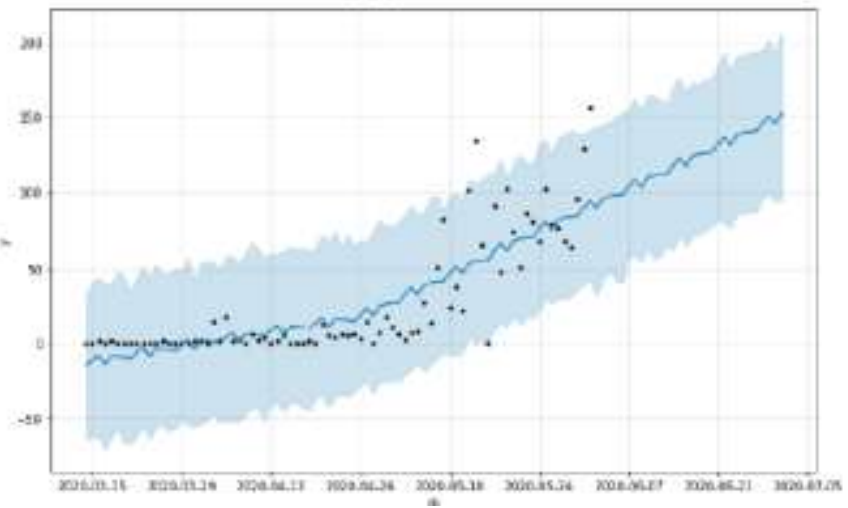

Figure 1: Future Forecast Using Prophet: 1. (a) Cumulative Forecast for India; 2. Daily Count Forecast for States: (b) Maharashtra, (c) Delhi, (d) Tamil Nadu, (e) West Bengal, ( $f$ ) Odisha

The daily data indicates that there are several spikes that reduce the model's predictability accuracy. The blue line represents the predicted value, as seen in Figures 1 (with uncertainty interval/ range) \& 2, and the red line/ black dots represent the actual value in Figures $1 \& 2$. From the figure, we can note that prediction accuracy is distinctly high, confirming the use of more robust nonlinear techniques, such as deep learning, exploits the non-linear characteristics of data and in the current pandemic, the scenario can serve as a reliable forecasting technique to predict cumulative and daily COVID-19 cases.
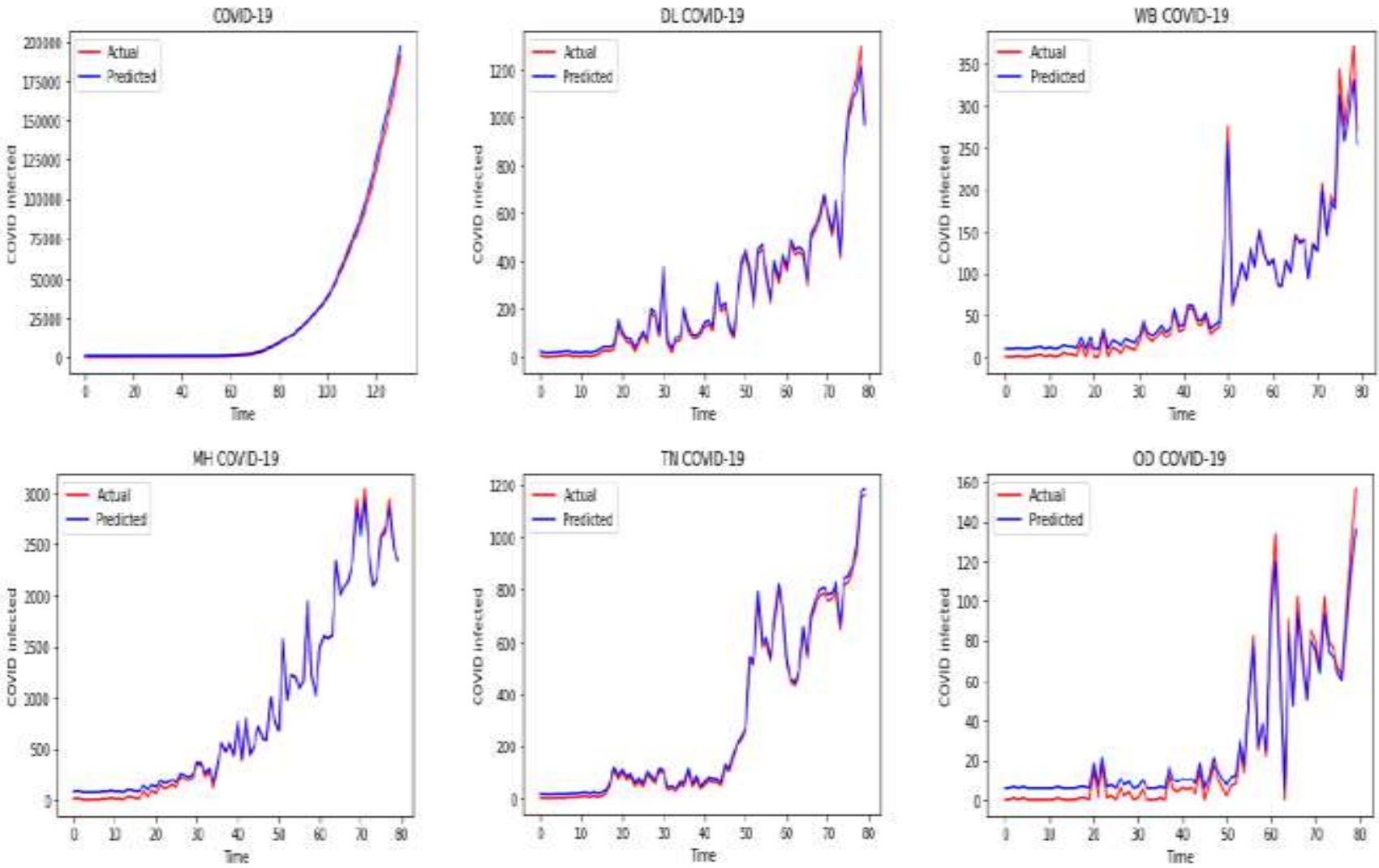

Figure 2: Prediction using GRU: 1. (a) Cumulative for India; 2. Daily Count for States: (MH) Maharashtra, (DL) Delhi, (TN) Tamil Nadu, (WB) West Bengal, (OD) Odisha

Therefore, if there are more fluctuations/ sudden spikes in daily new cases every day then the overall number of infected people can increase and there will be a further delay in obtaining curve flattening. The fluctuations/ sudden spikes cause non-stationarity in the dataset making it more difficult to predict exactly for regression models. Yet we can assume that if the spikes can be regulated with strict physical distancing and containment steps in the near future then curve flattening can be accomplished by the end of September. 


\section{IMPACTS OF COVID-19 on People, Planet and Profit of India}

COVID-19 is the infectious disease caused by the most recently discovered coronavirus. The World Health Organization (WHO) declared 2019-20 coronavirus episode a Public Health Emergency of International Concern (PHEIC) on 30 January 2020 and a pandemic on 11 March 2020.

The COVID-19 pandemic is considered as the most critical public health calamity of the century and the greatest challenge mankind has encountered since World War II. The COVID-19 pandemic has affected approximately 11.7 million people worldwide. As this research article is being written, Coronavirus Cases in India stand at 771,833, No Deaths reported 21,174 and 477,685 recovered

The Government of India adopted various measures to stop the spreading of the virus such as, Creating a toll-free helpline number, travel and entry restrictions. (International and Domestic), Closures and curfews, Lockdown, Evacuation, Tests for travelers etc.

On 30 January 2020, the first case of a coronavirus pandemic in India in 2019-20 was identified. In Kerala, registered its initial three incidents, each of which were students from Wuhan, China. [17]. As there is a rapid surge in the number of cases with each passing day, the impact of the pandemic is conspicuous across all the dimensions. This crisis has not only had an impact on people's well-being but also affected the environment and economy in a number of ways.

\subsection{Impact on planet}

The lockdown has also brought in some of the most vital environmental changes which never really took place in our living history. We have always been so hesitant towards protecting and revitalizing our Mother nature and its beauty. This lockdown has made most of us understand how our actions can have an impact on the natural environment. The 'Janata Curfew' was held on March 22, after which there was a significant dip in air pollution levels across the country. The air quality indices of some of the most polluted cities of India like Delhi, Mumbai, Lucknow, Kolkata and Bengaluru were staying within two digits.

Delhi air quality saw improvement as there was 30\% reduction in fine particulates because of COVID-19 mitigation measures as reported by System of Air Quality and Weather Forecasting and Research (SAFAR) [18]. Skies in the national capital turned an Azure blue and the air became breathable due to a 49 percent drop in the Air Quality Index.

The pandemic has caused a worldwide decrease in financial action and keeping in mind that this is a huge reason for concern, apparently the inclining down of human action has had a positive natural effect. Outflows and effluents from industry and transport have been diminished, and quantifiable information bolster poison clearing in air, soil, and water. Water bodies have additionally been cleared and the waterways Ganga and Yamuna have seen huge improvement in the water quality as indicated by the Central Pollution Control Board (CPCB).

Some positive impacts on the wildlife have also been observed since the enforcement of a nationwide lockdown. Nature and wildlife have replenished itself. Bombay Natural History Society reported a 25\% increase in flamingo migration since 2019,[19].

\subsubsection{Impact on air quality}

In a study carried out by Kumari and Toshniwal [20], the air quality data of three different cities in India, namely Delhi, Mumbai and Singrauli was analyzed. This data has been generated from Central Pollution Control Board's (CPCB) continuous air quality monitoring stations. The air quality data for air pollutants like PM10, PM2.5, NO2, SO2 and $\mathrm{O} 3$ prior to lockdown and post lockdown was studied for understanding the effect of the lockdown on the ambient air quality of these cities.

A significant reduction in concentration of particulate matter was observed for Delhi and Mumbai. In Delhi, PM10 and PM2.5 concentration reduced by 55\% and 49\% respectively. The city of Mumbai showed a similar trend with the reduction of $44 \%$ and $37 \%$ for coarse and fine particulate matter respectively. In terms of gaseous pollutants, NO2 concentration decreased by $60 \%$ and $78 \%$ for Delhi and Mumbai respectively. Mumbai showed higher decline (39\%) in ambient SO2 levels as compared to that for the city of Delhi (19\%). However, the ambient ozone concentration increased by $37 \%$ in Delhi and by $21 \%$ in Mumbai. The 
probable reason for the increase could be less consumption of ozone by titration reaction which could in turn be related to lower NOx concentrations in the ambient air. In contrast to these two mega cities, Singrauli has shown increase in the concentration of all the pollutants (except NO2), because of the coal based thermal power plants which were operational even during the lockdown phase.

\subsubsection{Impact on water quality}

Several news articles and the news reports on television have stated that the water quality of number on Indian rivers, namely Ganga, Yamuna etc. has improved. The concentration of Dissolved Oxygen (DO) in the water samples of river Ganga have been reported to be as high as $8 \mathrm{ppm}$ and Biochemical Oxygen Demand (BOD) which a main indicator of organic pollution has gone down to less than $5 \mathrm{ppm}$ at locations such as Kanpur and Varanasi [21]. The main reason that can be attributed to this improvement in the river water quality is the absence of any industrial effluent being discharged into these water bodies. Some of the other factors which are responsible for such an improvement include drastic reduction in otherwise commonly observed religious activities like worshiping, bathing and cremations. These activities have been temporarily banned [22]. The analysis of continuous water quality monitoring data for river Ganga has revealed that out of the total 36 monitoring stations, the water quality at 27 stations was found within the prescribed limits of Designated Best Use standards for the propagation of wildlife and fisheries and bathing [23].

Most parts of river Yamuna in Delhi are appearing clearer, blue and pure after years. The industrial wastes containing detergents and chemical mixtures which were creating a toxic foam have now vanished from Kalindi Kunj, Southeast Delhi [22].

\subsubsection{Impact on waste generation}

It has been estimated that the quantity of biomedical waste (BMW) generated in the last 2 months since lockdown in the city of Gurugram has shown a 40-fold rise [24]. In Ahmedabad, the quantity of BMW generated on a daily basis was $550-600 \mathrm{~kg} /$ day under normal circumstances. It rose to 1000 kilograms per day during the first phase of lockdown. It has been estimated to further rise up to 3000 kilograms per day with the increased usage of masks and other waste [25].

In terms of Municipal Solid Waste (MSW), the lockdown has resulted in an approximate reduction of 20$40 \%$ in the quantity of solid waste reaching the landfill or open dumping sites. In the city of Rajkot, a drastic reduction of more than $80 \%$ has been reported. This may be due to closure of industries which ultimately resulted in the migration of the workers working in those industries. Under normal circumstances, in Delhi city, the quantity of waste going to the dumpsite ranges from 3000 to 3700 metric tonnes per day. Due to the lockdown it showed significant reduction and amounted for 1800-2500 MT/day [24].

\subsubsection{Impact on noise levels:}

In India, airports/bus-stands/train-stations handle around 7800 flights daily, approximately 528,333 buses along with around 13,452 passenger trains and almost 9141 cargo trains run daily, respectively [26]. The analysis of noise data generated from five continuous noise monitoring stations across the city of Kolkata showed 50 to $75 \%$ noise reduction during the lockdown [27]. The noise level at some of the areas of Delhi showed a reduction of 40-50\% [28]. The data shows that from four metro cities of India before and during the lockdown period show 15-25\% decrease in the noise levels [29].

\subsection{Impact on People:}

The pandemic's impact is apparent in all sectors globally, but its influence in India has been tremendous, especially on the vulnerable groups, women and children. Households that were already unsafe together with families living in poor and marginal environments contributed to social inequities including gender-based violence and child abuse, lack of income, health facilities and other services. Financial shortages in these families have contributed to a condition where women continue to ignore their own interests while prioritizing family life and the requirements of other members at home. Many children below the age of 14 are being forced to use every means to earn some money for their survival. The risk of child trafficking has also risen from financial distress. This will create opportunities for the traffickers to acquire children for commercial sexual exploitation, child labour and other related crimes. Therefore, concerns such as menstrual hygiene, emotional wellbeing, education, safety and security are not in the priority list 
Societies, migrants or otherwise, have lost their livelihood and economic poverty has mainly affected communities all over. As indicated by government measurements, consistently in excess of 9,000,000 transient laborers move from India's country zones to huge populace habitats to look for some kind of employment at building destinations or plants, sending cash back to the places where they grew up and towns. A lot of these workers have set out on long journeys back to their native places on foot as without money and with the trains shut, they have no other option.

Due to the large influx of migrant workers, another major challenge for the state authorities is to ensure their rehabilitation in terms of livelihood and income security [30].

Several unplanned lockdown extensions across the country have also rendered things more challenging for these marginalized groups to seek some help or support with these issues. However, on the other hand, there are people who are taking this quarantine to relax and introspect their own selves. Many have been recognizing and working on their interests quite productively, whereas others still are exploring what they are passionate about.

The spread of the disease has affected the peoples mentally and psychologically. The disease has attracted the undivided attention of the people regarding the future due to the disease.

\subsection{Impact on Profit:}

The lockdown procedure has been proceeding for as far back as scarcely any months and has just hindered the supply side, quickening the stoppage further and imperiling the financial prosperity of millions. At first, the government had lockdown transport services, shut all open and private workplaces, production lines and limited assembly. In light of late investigations, a few financial experts have said that there is a vocation loss of 40 million individuals in the nation, the vast majority of which are in the unorganized sector. The predictions of India gradually going into recession has already affected the unorganized sector and also a large number of semi-skilled jobholders who have and are on a verge to losing their employment. Travel limitations and quarantine is influencing a large number of individuals who have left Indian production lines due to shortage of labour, just-in-time supply chains and triggering sales warnings across technology, automotive, consumer goods, pharmaceutical industries and different enterprises.

However, as businesses close to help prevent the transmission of COVID-19, financial concerns and job losses are one of the first human impacts of the virus.

Estimated quarterly impact from the coronavirus (COVID-19) on India's GDP growth in from April to June, 2020 is $-9.3 \%$ (31) As of May 6, 2020, India's government deployed some 348 billion Indian rupees to combat the effects of the coronavirus(COVID-19)lockdown (32) .The IMF is anticipating an dramatic decrease in India's GDP growth rate to $1.9 \%$ by 2020, with a rapid v-shaped rebound to $7.4 \%$ by 2021 (33)

The International Monetary fund (IMF) has stated that the economic impact the coronavirus breakout might have on the world's economy, and hence people's livelihood, might supersede the impact of the 2008 Financial Crisis. Decrease in demand, in turn, happens due to lower ability of people to spend money. We will witness, in the future, spikes in poverty, unemployment and worldwide hunger. That could also lead to an increasing trend in black markets. Industrialists and employers will also bear the brunt of the crisis as they will lack the funds to pay their employees. Hence, people from all walks of life will have to face the consequences of the economic crisis in some way.

\section{Conclusion}

After many attempts to improve the model's performance, the predictor has provided reliable results. But due to considerable changes over time in the disease's spread pattern in India due to various factors, such as different Indian people's movement patterns at different lockdown times, large-scale labor migration, the shift in diagnostic facilities, coronavirus evolution, and so on will make it difficult for predictive models. These triggers are far beyond any prediction model's influence. The pattern clearly shows the disease will remain in the country for several months to come. It will not leave us in a comfort zone, but the fact must be accepted, and appropriate plans must be made to address all the pandemic outbreak-related issues. Even though India faces an economic and health crisis the governance and key policy will help the country fight against and overcome this unprecedented challenge. 


\section{References}

[1.] World Heath Organisatioin, 2020. Coronavirus disease (COVID-19) pandemic, https://www.euro.who.int/en/health-topics/health-emergencies/coronavirus-covid-19/novelcoronavirus-2019-ncov

[2.] Anderson, R.M., Heesterbeek, H., Klinkenberg, D., Hollingsworth, T.D., 2020. How will countrybased mitigation measures influence the course of the COVID-19 epidemic? Lancet 395, 931-934.

[3.] [S. Mallapaty, What the cruise-ship outbreaks reveal about COVID-19, Nature 580 (7801) (2020) . $18-18$

[4.] K. Liu, Y. Chen, R. Lin, K. Han, Clinical features of COVID-19 in elderly patients: a comparison with young and middle-aged patients, J. Infect. (2020).

[5.] Debanjan Parbat, Monisha Chakraborty, A python-based support vector regression model for prediction of COVID19 cases in India, Chaos, Solitons \& Fractals, Volume 138, 2020, 109942, ISSN 0960-0779, https://doi.org/10.1016/j.chaos.2020.109942.

[6.] Stefano Boccaletti, William Ditto, Gabriel Mindlin, Abdon Atangana, Modeling and forecasting of epidemic spreading: The case of Covid-19 and beyond, Chaos, Solitons \& Fractals, Volume 135, 2020, 109794, ISSN 0960-0779, https://doi.org/10.1016/j.chaos.2020.109794.

[7.] Tobías, A., 2020. Evaluation of the lockdowns for the SARS-CoV-2 epidemic in Italy and Spain after one-month follow-up. Sci. Total Environ. 725, 138539 (in press).

[8.] S. Tuli, N. Basumatary, S.S. Gill, M. Kahani, R.C. Arya, G.S. Wander, R. Buyya, HealthFog: an ensemble deep learning based smart healthcare system for automatic diagnosis of heart diseases in integrated iot and fog computing environments, Future Generat. Comput. Syst. 104 (2020) 187200.

[9.] R. Dey and F. M. Salem, "Gate-variants of Gated Recurrent Unit (GRU) neural networks," 2017 IEEE 60th International Midwest Symposium on Circuits and Systems (MWSCAS), Boston, MA, 2017, pp. 1597-1600, doi: 10.1109/MWSCAS.2017.8053243.

[10.] F. Gong, N. Han, D. Li and S. Tian, "Trend Analysis of Building Power Consumption Based on Prophet Algorithm," 2020 Asia Energy and Electrical Engineering Symposium (AEEES), Chengdu, China, 2020, pp. 1002-1006, doi: 10.1109/AEEES48850.2020.9121548.

[11.] Saadat, D. Rawtani, C.M. Hussain, Environmental perspective of COVID-19, Sci.Total Environ. 728 (2020), https://doi.org/10.1016/j.scitotenv.2020.138870138870.

[12.] K. Cho, B. Van Merriénboer, D. Bahdanau and Y. Bengio, "On the properties of neural machine translation: Encoder-decoder approaches" in arXiv:1409.1259, 2014, [online] Available: https://arxiv.org/abs/1409.1259.

[13.] Guizhu Shen, Qingping Tan, Haoyu Zhang, Ping Zeng, Jianjun Xu, Deep Learning with Gated Recurrent Unit Networks for Financial Sequence Predictions, Procedia Computer Science, Volume 131, 2018, Pages 895-903, ISSN 1877-0509, https://doi.org/10.1016/j.procs.2018.04.298.

[14.] Hochreiter, S. (1998). The vanishing gradient problem during learning recurrent neural nets and problem solutions. Int. J. Uncertain. Fuzziness Knowl.-Based Syst., 6(2), 107-116, https://doi.org/10.1142/s0218488598000094.

[15.] Chung, J., Gülçehre, Ç., Cho, K., and Bengio, Y. (2014). Empirical evaluation of gated recurrent neural networks on sequence modeling. CoRR, abs/1412.3555.

[16.] Facebook Open Source. Prophet, Forecasting at scale, https://facebook.github.io/prophet/

[17.] Balasubramanian Arul and Ramalingam Kothai, Int. J. Res. Pharm. Sci., 2020, 11(SPL)(1), 715,2019 Novel Coronavirus: A mysterious threat from Wuhan, China-A current review https://doi.org/10.26452/ijrps.v11iSPL1.1975)

[18.] The Hindu. Air quality improves amid lockdown in India, https://www.thehindu.com/news/national/air-quality-improves-in-india-amidlockdown/article31234638.ece ;2020 [accessed 03 April 2020].

[19.] Hindustan Times, 2020. 25\% more flamingos in MMR, https://www.hindustantimes.com/mumbainews/25-more-flamingos-in-mmr/story-OOBiPNb9rTsInMEAli8e2L.html ;2020 [accessed 20 April 2020]. 
[20.] Kumari, P., and Toshniwal, D. (2020). Impact of lockdown measures during COVID-19 on air quality- A case study of India. International Journal of Environmental Health Research. DOI: 10.1080/09603123.2020.1778646

[21.] SANDRP. Ganga-Yamuna-Cauvery Flow Cleaner in Lockdown: what Can We Learn?" DRP News Bulletin, South Asia Network on Dams, Rivers and People (SANDRP). Retrieved from: https://www.sandrp.in/2020/04/06/drp-nb-6-april-2020-ganga-yamuna-cauvery-flow-cleaner-inlockdown-what-can-we-learn/\#more-34730;2020 [accessed 12 April 2020]

[22.] Lokhandwala, S., and Gautam, P. (2020). Indirect impact of COVID-19 on environment: A brief study in Indian context. Environmental Research 188, 109807, 1-10.

[23.] The Tribune. Lockdown Helps Improve Health of Ganga, https://www.tribuneindia.com/news/nation/lockdown-helps-improve-health-of-ganga-64936;2020 [accessed 04 April 2020]

[24.] Somani, M., Srivastava, A., Gummadivalli, S., Sharma, A. (2020). Indirect implications of COVID-19 towards sustainable environment: An investigation in Indian context. Bioresource Technology Reports, 11, 100491, 1-10.

[25.] Times of India. 350 tonnes of trash disappear from streets of Ahmedabad. http://timesofindia.indiatimes.com/articleshow/75039037.cms?utm_source=contentofinterest\&utm _medium=text\&utm_campaign $=$ cppst $; 2020$ [accessed 09 April 2020]

[26.] Business Standard. Airports prepare to handle $30 \%$ flights in first phase of ops after lockdown, https://www.business-standard.com/article/companies/airports-prepare-to-handle-30-flights-infirst-phase-of-ops-after-lockdown-120042901409_1.html ;2020 [accessed 30 April 2020]

[27.] Basu, J. (2020). COVID-19: Sounds of Birds Replace Noise Pollution in Kolkata. Down to Earth. Retrieved from. https://www.downtoearth.org.in/news/environment/covid-19-sounds-of-birdsreplace-noise-pollution-in-kolkata-70241.

[28.] Time of Inida. Covid-19: Noise pollution falls as lockdown rings in sound of silence, https://timesofindia.indiatimes.com/india/covid-19-noisepollution-falls-as-lockdown-rings-insound-of-silence/articleshow/75309318.cms ;2020 [accessed 24 April 2020]

[29.] Somani, M., Srivastava, A., Gummadivalli, S., Sharma, A. (2020). Indirect implications of COVID-19 towards sustainable environment: An investigation in Indian context. Bioresource Technology Reports, 11, 100491, 1-10.

[30.] Srirang K Jha and Manoj Kumar COVID 19 Scare and Plight of Indian Migrant Workers: Decoding the Impact of Nationwide Lockdown, Journal of Politics \& Governance Vol. 8 No. 4, April 2020, Pp. 8-11 ISSN: 2278473X (Print) 2456-8023 (Online))

[31.] Statista. Impact of the coronavirus (COVID-19) on the Indian economy - statistics \& facts, https://www.statista.com/topics/6304/covid-19-economic-impact-onindia/\#dossierSummary__chapter1) ;2020 [accessed 24 June 2020]

[32.] Statista, 2020. Value of government aid towards the coronavirus (COVID-19) across India as of May 6, 2020, by type, https://www.statista.com/statistics/1112551/government-aid-value-forcoronavirus-india/);2020 [accessed 14 May 2020]

[33.] Institute for Sustainable Development and International Relations (IDDRI), 2020. The impacts of COVID-19 on India: risk factors, sustainable development and climate action, https://www.iddri.org/en/publications-and-events/blog-post/impacts-covid-19-india-risk-factorssustainable-development-and ;2020 [accessed 24 April 2020] 